\title{
Computer control of memory experiments on a large-scale timesharing system
}

\author{
MICHAEL FRIENDLY and PAT FRANKLIN \\ York University, Downsview, Ontario M3J IP3, Canada
}

\begin{abstract}
This paper describes a computer system for running verbal learning and memory experiments using a large-scale timesharing computer. Its application is illustrated by control programs used to set up, execute, and analyze a series of interactive free recall experiments. While limitations of timesharing systems for experimental control surely exist, they can often be removed by simple hardware or software. Further, a large machine can provide significant advantages in cost and software development over dedicated laboratory minicomputers. It is possible to obtain the advantages of both types of systems by introducing local intelligence to provide more precise timing and flexible control of experimental devices, while retaining the power and hardware and software resources of the large machine.
\end{abstract}

There are a variety of alternatives for experimenters considering automated research. Possible systems include single-user micro- and minicomputers (Murdock, Dufty, \& Okada, 1972), multiuser micros and minis (Santa \& Streit, 1978; Scholz \& Halff, 1974), networks of small computers (Wallace, Johnson, \& Johnson, 1978), general departmental computer facilities (Fitzhugh \& Glaser, 1975; Polson, 1978; Proudfoot, 1978), and general university-wide installations (Huesmann, 1969; Hunt, 1969; Potts, 1976).

Each of these systems represents a different level of cost and personal involvement in system development. Obviously, initial expenditures are important in this decision, but there are other equally important factors. For example, a microcomputer system that costs $\$ 3,000$ and takes 2 man-years to learn and put in to use is more expensive than a $\$ 15,000$ system that can be installed and operationalized within 1 month. A system that comes complete with a variety of high-level language processors, utilities, and software tools is likely to be cheaper in the long run than a higher priced system with limited software. Indeed, as hardware costs continue to drop, making minis and micros more available, many users are finding that the limited availability of general software (e.g., operating systems, text editors, file systems and utilities, language translators) is a limiting factor in computerized experimentation (Bowles \& Hollan, 1978; Polson, 1978; Scholz, 1978).

This work was supported by National Research Council of Canada Grant A8615 to the first author. We are grateful to Stephen Madigan, who provided us with a listing of an experimental control program from which our system grew. Richard Levine, Serge Medow, and John Meskes provided programming support at various stages of the project, and David Hoffman assisted with documentation and the preparation of this paper. We also acknowledge with gratitude the cooperation and special assistance of Hal Anderson, Tony Pierre, Ken Tulino, and other staff members of the York University Department of Computer Services.
This paper describes the stage-wise development of a computer facility for running memory and learning experiments with adult human subjects, based on a topdown approach to developing computer control. This system was originally implemented on a large universitywide timesharing computer. Our strategy has been to try to obtain maximum utility from a fixed level of involvement by using the available hardware and software resources of the large machine wherever possible at any stage; as stated by Kernighan and Plauger (1976), we "let someone else do the hard part." This approach allows us to focus our efforts and somewhat limited financial resources on those components most crucial to automating experimental control.

We discuss the first stage of this development mainly in terms of control programs used to set up, execute, and analyze a series of interactive free recall experiments. The system has been operational for 3 years and has been used to run a variety of memory experiments, including free recall, item recognition, lexical decision, and category verification tasks. Some aspects of its present status and planned future development are touched on briefly.

\section{HARDWARE}

\section{Central-Site Computer}

Our experimental control system runs on a moderatesize DEC-10 installation, a KI10 36-bit processor with $256 \mathrm{~K}$ main core, hardware double-precision arithmetic, and a typical complement of disk (2-RP04 and 2-RP06) and tape drives (2-TU70), readers, and printers. A communication link to an IBM $370 / 158$ provides twoway remote job submission as well as protected access to programs and data files between machines. The DEC-10 system supports remote batch, real-time jobs, priority timeslicing, and networking of remote stations. Subject to approval of the York University computer 

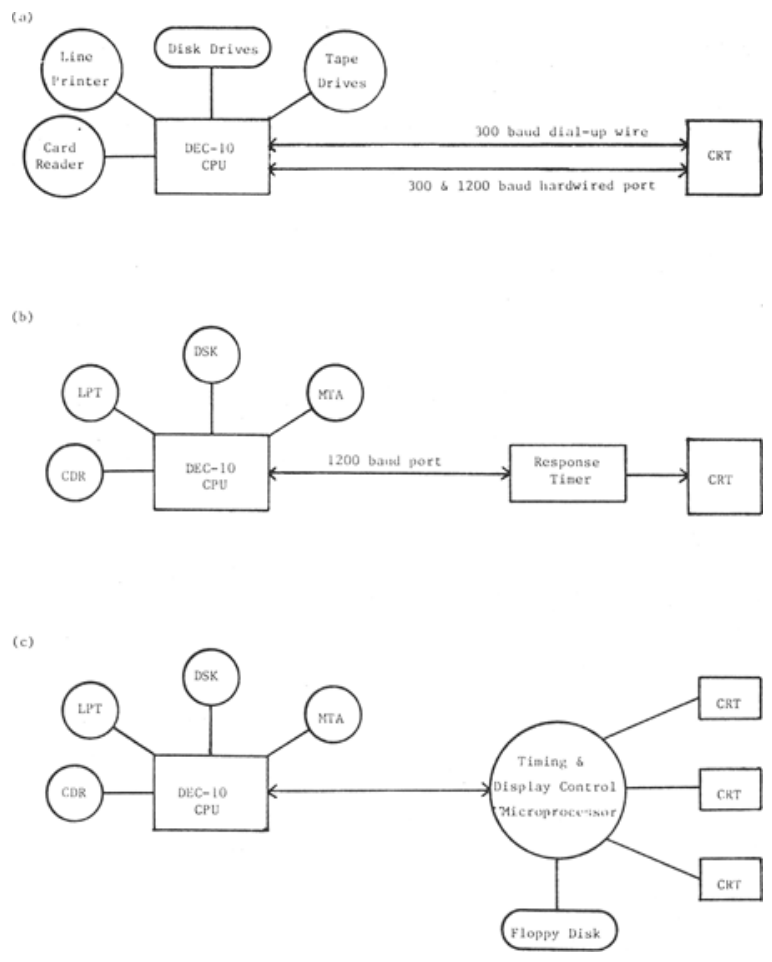

Figure 1. Stages in developing a timeshared computer by adding local intelligence.

center, small $\mathrm{I} / \mathrm{O}$-bound jobs may be locked in core and run at top priority.

The software available through this system includes a wide variety of high-level language processors, a macroassembler, the SOS/RUNOFF text-editing system, and a macro-interpreted command language (MIC) that provides access to all system parameters associated with a given job.

\section{Local Hardware}

The components of the initial system are shown schematically in Figure 1a. Our investment at this stage was a Datamedia Elite 1520 CRT display terminal $(\$ 2,200)$ with a dual (ASCII/APL) character set and programmable cursor control. It is connected to the DEC-10 by both a 300 -baud dial-up line and a 1,200 baud hardwired port. Later, a Polytronics universal response timer $(\$ 600)$ was added for reaction time experiments (Figure 1b). The timer can be connected through the key board of the terminal or interfaced to simple microswitch response boxes or panels (see Potts, 1976).

\section{THE IFR SYSTEM}

The IFR system grew from a need to automate contingent presentation in free recall, in which the stimulus presentation on Trial $\mathrm{N}+1$ depends on some characteristic of the subject's response on Trial N. The system consists of modular software designed to completely automate the running of a free recall experiment. That is, the system as a whole provides facilities for setting up an experiment, running subjects, and analyzing data. The components of the system (programs and files) are shown schematically in Figure 2.

\section{Word Pools and Stimulus Selection}

In memory experiments, subjects are presented a set of stimulus items selected from some larger pool. The stimulus items are usually words, but in general, they consist of any string of ASCII characters, including control characters. This allows a character-oriented interface (Fitzhugh \& Glaser, 1975), in which new stimulus presentation devices can be added or changed easily without affecting the overall design of the system.

A "word pool" consists of a series of stimulus items, as defined above. Associated with each stimulus item there may be scale values for that item on one or more variables (e.g., word frequency, concreteness, imagery) used in selecting items for a given subject. The IFR system selects items randomly within a range on these variables (specified for each condition), so that every subject receives a unique list. A word pool is stored as a direct-access file with a record for each item, containing the character representation of the item and its measured scale values. At present, word pools include, among others, the Battig and Montague (1969) norms of categorized words and the Paivio, Yuille, and Madigan (1968) norms.

Taking advantage of the wide choice of languages available on the large machine, we wrote the control programs for the IFR experiments in APL (Iverson,

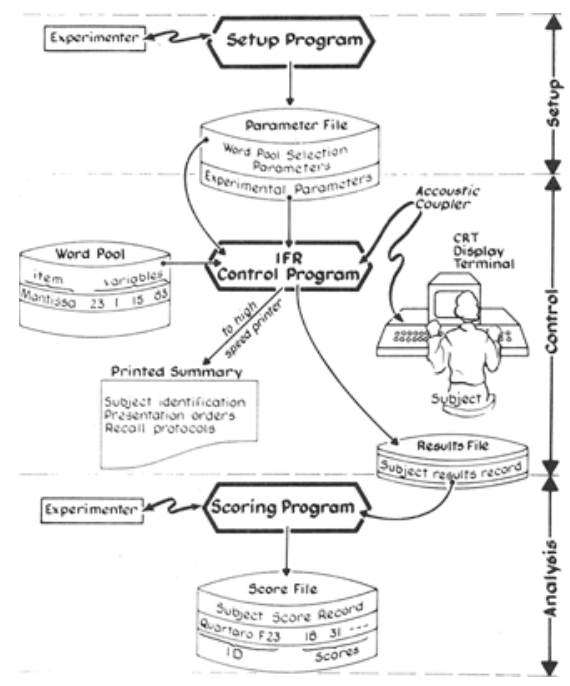

Figure 2. Design of the IFR system for experimental control. SETUP, CONTROL, and ANALYSIS indicate the operations performed during the corresponding phases of an experiment. 


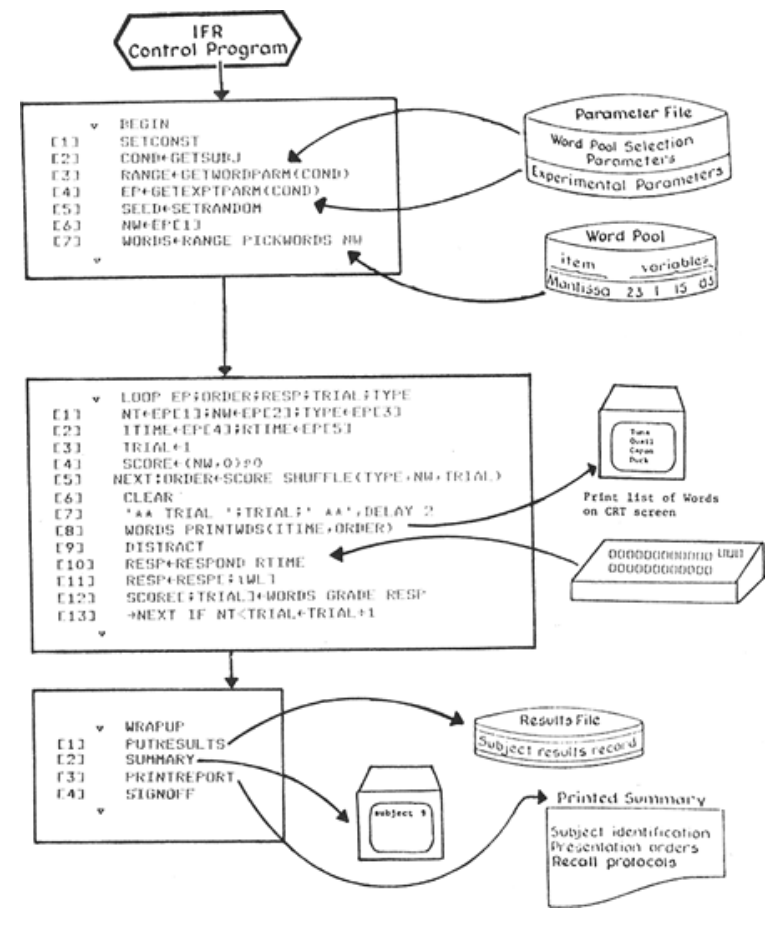

Figure 3. The main blocks of the IFR control program. BEGIN initializes experimental parameters (EP) and word selection ranges and then randomly selects a list of NW words. LOOP executes NT recall trials with a presentation time of ITIME sec/item and RTIME sec allowed for recall. WRAPUP prints and saves the results. The functions called by BEGIN and LOOP are shown in the Appendix.

1962) and selected FORTRAN for scoring the results and data analyses (Friendly, 1975).

Figure 3 shows the flow of experimental events in IFR and the interaction of software and hardware. In free recall, the subject is given a series of trials in which a list of words is displayed, and then he is required to recall as many of these words as he can. This structure is mirrored in the IFR control programs in LOOP over trials that form the core of the programs. BEGIN is responsible for initialization procedures and WRAPUP saves the subject's data on disk, provides feedback, and sends a complete protocol of the session (presentation details, recall results, and various summary measures) to the high-speed printer.

In the program a clear separation is made between the logic of the experimental events and possible parametric variations. That is, the variable features of the experiment, such as the number of words, number of trials, times for presentation and response, and so on, are read by the CONTROL program from the parameter file during initialization (BEGIN). The parameter file also identifies the word pool to be used with a specific experiment, and it contains the variables that govern the selection of stimuli from the pool. Thus, no changes to the CONTROL program are required to set up a specific IFR experiment, and the parameter files can be constructed by an interactive SETUP program. In this way, the same software can be used by students in laboratory courses to design and run small automated experiments as part of their course work.

\section{Data Management}

The large machine is particularly valuable for data management. We maintain a number of large stimulus pools, used across many experiments, and a set of parameter and results files associated with each experiment. As the volume of such data grows, it is crucial to be able to access selected information quickly and easily. One solution to this problem is to develop a comprehensive data-base management system (e.g., Durrett, 1976) for experimental control and data analysis.

However, on the DEC-10, we have again found it possible to take advantage of existing resources. At one level, the operating system provides hierarchical file directories, which simplifies managing many files. At a lower level, the file I/O facilities of the DEC-10 APLSF implementation go a long way toward meeting our needs.

For stimulus pools and parameter files, the use of direct-access files makes stimulus selection quite easy. For example, in APL the instruction,

$$
D \leftarrow \boxminus[C] \text { INFILE }
$$

reads component $\mathrm{C}$ (a variable-length record) from file INFILE and assigns its contents to a variable $D$.

The results files present a somewhat different problem, since a subject's data record may contain a heterogeneous collection of data items of varying length, type (character, integer, logical, real), and shape (scalars, vectors, and arrays), and these may vary considerably from one experiment to the next. Our results files are written in binary access mode in a selfdescribing format. For example, the instruction,

\section{A $⿴$ OUTFILE}

writes the data item A to file OUTFILE with a header of a few words that encodes the data type, length, and $\operatorname{rank}(0=$ scalar, $1=$ vector, $2=$ matrix, etc. $)$. Since this information is contained in the file itself, most of the "hard part" of data management is done for us. Programs that access these files need to know the number of components in a subject's data record, but not the details of their composition.

The only drawback of this scheme is that the data files cannot be read directly by standard packaged programs. Still, it is relatively easy to write a small utility program to preprocess the results files for standard analysis packages. The results from most 
experiments need to be preprocessed anyway, to cull bad data records, perform preliminary transformations, or calculate various derived measures from the raw results, so this has not been any burden.

\section{EVALUATION}

\section{Virtual Apparatus}

In interfacing stimulus and response devices, there is often a tradeoff between hardware and software, so that many component operations in an experiment can be carried out under software control from the central machine or by acquiring a special-purpose device.

To make the timesharing computer more versatile, the subject I/O station is regarded as a "virtual apparatus." The physical device at the subject station (a CRT with cursor addressing, for example) has a certain range of basic sensorimotor I/O capabilities. A virtual apparatus, like its physical counterpart, is composed of components, but many are fashioned in software rather than hardware. Within limits, we can design, build, and test a virtual apparatus far more easily and cheaply than we can construct a physical one (see also, Vesonder, Lesgold, \& Wolf, 1978). Once it works, we can then evaluate its limitations and make it work better or faster by introducing specialized hardware.

\section{Timing}

For example, the difficulty in timing stimulus and response events with sufficient precision is probably most often cited as an inherent limitation of large-scale timesharing systems for computerized research due to the probability of being swapped out of core at any instant.

However, timing is always a matter of degree, in relation to demands of particular tasks. We need to obtain response times to millisecond accuracy and insure that stimulus display times are precise to within 3\%-5\%. (Typical durations range from $400 \mathrm{msec}$ to 10 or $20 \mathrm{sec}$ per item.)

We have found a solution to the timing problem that more than adequately meets our needs, although it might not prove satisfactory for very brief stimulus durations. The Polytronics response timer handles all the response timing transmitting a one-character response followed by a four-digit time value, encoded as an ASCII string. This timer is relatively inexpensive $(\$ 600)$, but in our present system, one must be provided for each subject station.

We were able to obtain high-priority privileges, so we decided to solve the problem of presentation duration in software. Small FORTRAN and MACRO programs were developed to be locked in core and executed at high priority to display stimulus information. On-times and delays are provided by writing idle characters, and at 1,200 baud, these times can be resolved to within $8 \mathrm{msec}$.

\section{Cost}

While the top-down approach to development reduces costs on hardware purchasing, there are concealed costs for developing software and using university equipment. The software has to be developed regardless of the approach to automation. However, the cost of the timesharing per connect hour can seem high. In our particular location, these costs have worked out at approximately $\$ 8 / \mathrm{h}$ (CPU time plus connect time) during the last 3 years. In the early stages of developing a computer facility, much of the time the equipment is not used to capacity. If the user owns the hardware, then he must pay maintenance on it regardless of whether it is fully operational. However, if someone else owns the expensive hardware, then the user only pays when he is connected and using the system.

\section{Programming Languages}

We chose APL as the language to develop experimental control programs because of its extensive capabilities for manipulating character strings and data arrays. Although it is an interpretive language, and therefore somewhat inefficient at execution time, it is highly efficient in terms of programming time.

For comparison with other languages developed for computer control (McLean, 1969; Pinkus \& Gregg, 1973; Schòlz, 1973), Figure 4 shows the basic Sternberg (1966) item recognition paradigm, programmed in APL. The program chooses digits at random for presentation and presents varied sets within a range of set sizes (one to five), with a .5 probability of a positive probe. Our item recognition programs are much lengthier, since they include options for stimulus type, and parametric values such as intertrial interval. They also include functions for data storage and hard-copy output, including on-line analysis of subject performance indices.

To demonstrate the more sophisticated capabilities of APL, Figure 5 shows the IFR spelling algorithm that checks subjects' responses during recall trials for misspellings and typographical errors. The program calculates a correlational value between a typed response word and each word in the experimental list, based on letter matches and positional proximity. In a recent assessment, a sample of 1,837 responses from several

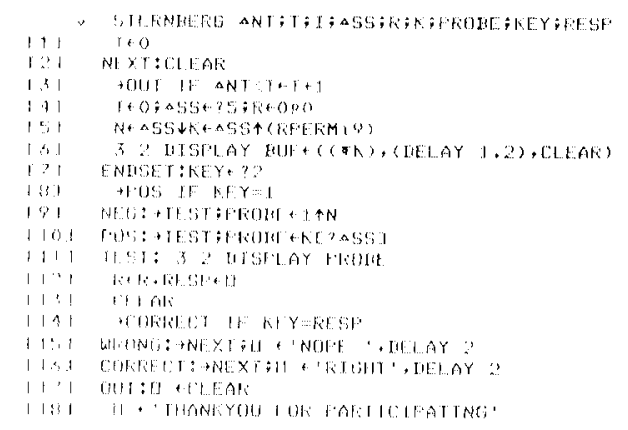

Figure 4. Stemberg item recognition program. 

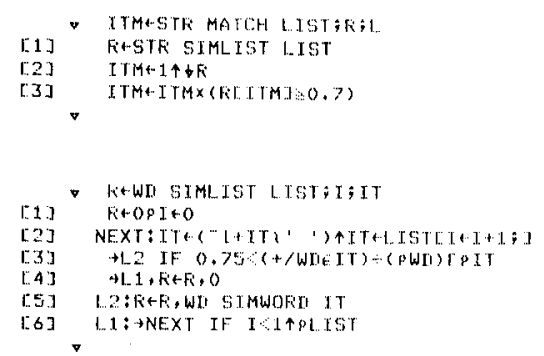

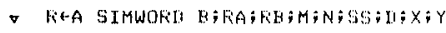

R WORI SIMILARITY ALGOFITHM

RAOPAOKETE

$\rightarrow O$ IF $0=F+t / A E E$

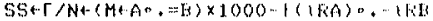

$M+N=Q$ (RH, FA) $P(R A \times F B) \rho S S+0=5$

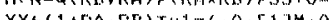

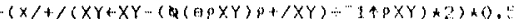
$\mathrm{R}+\mathrm{S} \times(\mathrm{R} \div \mathrm{FIAI} \mathrm{RB}) \star 0+5$

Figure 5. Spelling correction algorithm used for typed recall. SIMWORD computes a correlation-like statistic for the similarity of two words, A and B. SIMLIST uses SIMWORD to find the similarity between a response, WD, and a word list, LIST. The similarity calculation is only made for list words that have at least $75 \%$ letters in common with WD. MATCH accepts WD as correct if the maximum correlation is at least .7.

experiments contained 161 misspellings, of which 1 was misclassified by the algorithm. An equivalent program in FORTRAN (or similar language) would be over 90 lines of code, and the computations would severely strain many small systems.

At the same time, there are some experiments that are inefficient in APL, so we developed a set of low-level routines in MACRO and FORTRAN similar to those described by Proudfoot (1978). In the DEC-10 APLSF implementation, these can be called from APL, so the block functions need not be rewritten.

\section{Future Development}

The IFR system and other systems that evolved from it represent the first stage in developing a computerized laboratory in a step-by-step process. The next step is to introduce a small microcomputer as a front end to the large timesharing machine (Figure 1c). The local machine initially handles the timing and control of the stimulus and response devices and leaves the bulk of the work to the central machine. For example, the main frame can send a block of data to the micro that contains all stimulus presentation information sufficient for one trial. The micro would be responsible for interpreting the control information and for handling all device translation and timing. As the micro is integrated into the system, it can provide a basic timesharing system, serving several peripheral subject stations simultaneously. As this stage draws near completion, the programs are developed on the central computer, but run on the local machine. All of the lower level software developed in the early part of Stage 2 are dedicated to improving process control operations. At the end of Stage 2, one would have a mode of operations similar to a much larger departmental computercontrolled facility, at a small fraction of the cost.

\section{REFERENCES}

Battig, W. F., \& Montague, W. E. Category norms for verbal items in 56 categories: A replication and extension of the Connecticut category norms. Journal of Experimental Psychology Monograph Supplement, 1969, 80(3, Pt. 2).

Bowles, K. L., \& Hollan, J. D. An introduction to the UCSD PASCAL system. Behavior Research Methods \& Instrumentation, 1978, 10, 531-534.

DuRRETT. H. J., JR. A simple information management system for use in on-line experinentation. Behavior Research Methods \& Instrumentation, 1976, 8, 157-163.

Fitzhugh, R. J, \& Glaser, R. A general-purpose computer system for a research and development center. American Psychologist, 1975, 30, 205-211.

Friendiy, M. L. Computer processing of free recall data. Behavior Research Methods \& Instrumentation, 1975. 7. 47-50.

Huesmann, L. R. Controlling experiments with a general purpose time-sharing system. Behavior Research Methods \& Instrumentation, 1969, 7, 276-280.

Hunt, E. B. Conversational computing systems as laboratory instruments. American Psychologist, 1969, 24, 199-202.

lverson, K. E. A programming language. New York: Wiley, 1962.

Kernighan, B. W., \& Plauger, P. J. Software tools. Reading, Mass: Addison-Wesley, 1976.

McLean, R. S. PSYCHOL: A computer language for experimentation. Behavior Research Methods \& Instrumentation, 1969, 1, 323-328.

Murdocx, B. B., JR., Dufty, P. O., \& Okada, R. Using the PDP-12 in verbal learning and short-term memory research. Behavior Research Methods \& Instrumentation, 1972, 4, 70-71.

Paivio, A., Yuille, J. C., \& Madigan, S. A. Concreteness, imagery and meaningfulness values for 925 nouns. Journal of Experimental Psychology Monograph Supplement, 1968, 76(1, Pt. 2).

Pinkus, A. L., \& Gregg, L. W. APCOL: A programming system for computer-controlled psychological laboratories. Behavior Research Methods \& Instrumentation, 1973, 5, 165-172.

Polson, P. G. Microprocessors: Their impact on real-time computing in psychology. Behavior Research Methods \& Instrumentation, 1978, 10, 139-147.

Poтts, G. R. Use of a campus-wide timesharing computer to run reaction time experiments. Behavior Research Methods \& Instrumentation, 1976, 8, 179-181.

Proudfoot, R. G. YEPS: Running real-time experiments on a timesharing system. Behavior Research Methods \& Instrumentation, 1978, 10, 291-296.

SANTA, J. L., \& STREIT, P. A microprocessor reaction time laboratory. Behavior Research Methods \& Instrumentation, 1978, 10, 352-355.

Scholz, K. W. PROSS: A process control programming language. Behavior Research Methods \& Instrumentation, 1973, 5, 245-247.

Scholz, K. W. A floppy disk operating system for the 8080 and Z80. Behavior Research Methods \& Instrumentation, $1978,10,527.530$.

Scholz, K. W., \& HalfF, H. A decentralized computer network for supervision of multiple psychological laboratories. Behavior Research Methods \& Instrumentation, 1974, 6, 139-143.

STERNBERG, S. High speed scanning in human memory. Science, 1966, 153, 652-654.

Vesonder, G. T., Lesgold, A. M., \& Wolf, R. V. The use of microprocessors in a timeshared computer facility for research, development, and teaching. Behavior Research Methods \& Instrumentation, 1978, 10. 510-515.

Wallace, R., Johnson, R. N., \& Johnson, J. H. An introduction to microcomputer networking concepts. Behavior Research Methods \& Instrumentation, 1978, 10, 522-526. 


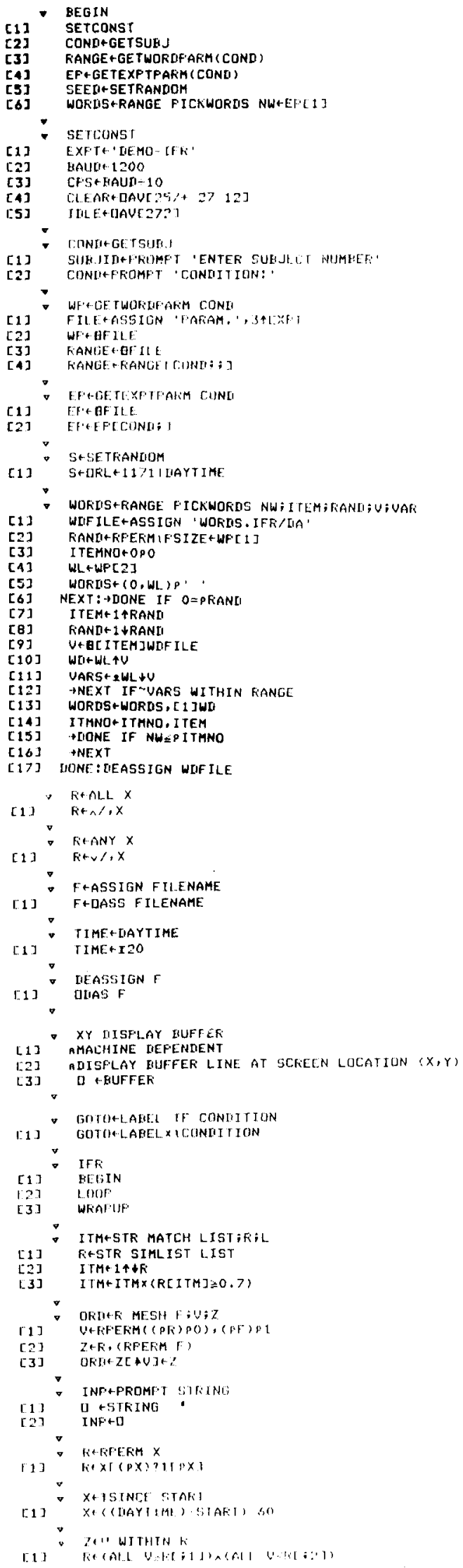

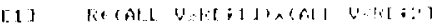

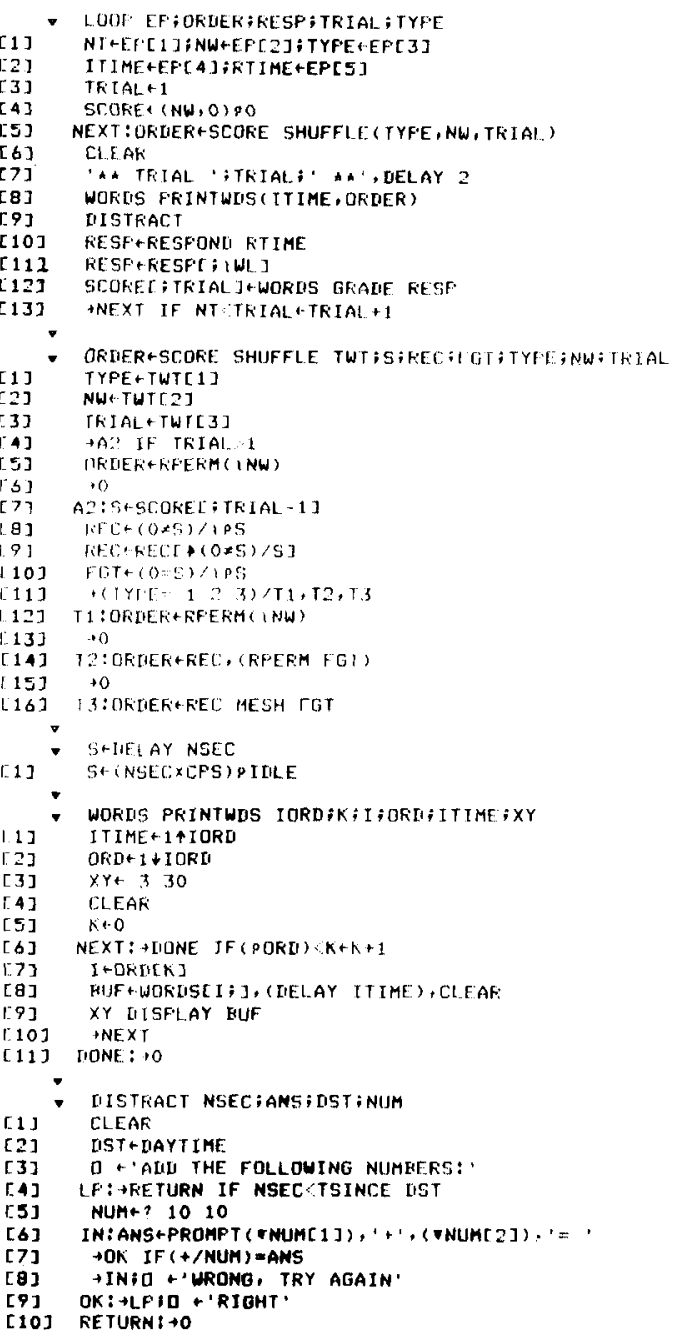

FU FESFONI FT IME; INIL ; NF; FESTART

$[1]$ CLEAR
$[2]$ D tha RECALL A .

[3] $\left[\begin{array}{ll}1+30 \\ \text { [. } 3]\end{array}\right.$

[4] RH'

[6] MORE: +OUER IF RTIME TSINCE FITART

[J] IN+D

[Q] PIONE IF INA,=",

[.9] $R \leftarrow R+L \uparrow I N$

[10] MOKE

[11] OULE: A 'FECALL FEFIOIT IS OVER'

[12] HONE:D WND OF TKIAL.

[13] NREt $(P F) \div 1$.

[1.4] Hit $(\mathrm{NH}, \mathrm{L}) \rho \mathrm{R}$

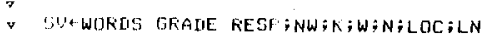

[1] NW: $1 \uparrow \rho W O F E S$

[2] SU. $(2+N W) 90$

[.] SU]

[4] NEXT: $\rightarrow 0$ IF $(19 P$

[S] WhESP[K;

[6] LOC.WOKISA. $=w$

I. 7$] \quad[N+L O C: 11$

[Q] HOUNI IF 1 ANY LOC

[9] CKSILLL: TFOUNA If OFLNEW MATCH WOROS

[-10] DNTF

[11] IOINIF; +CORKECT IF $0=$ SULINI

[12] 12$] \mathrm{KEE}$

[13] CHAFELT:SULLN]

[14] NEXI

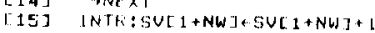

[.16] INFXT

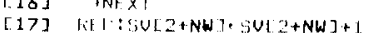

[18] kit.:5iju

$\mathrm{v}$

- IJKAFI

5.7 Ferrotels

127 summalir

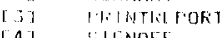

